\title{
Self-imaging dynamics in nonlinear GRIN multimode optical fibers
}

\author{
F. Mangini ${ }^{1, *}$, T. Hansson ${ }^{2}$, A. Tonello ${ }^{3}$, T. Mansuryan ${ }^{3}$, M. Zitelli ${ }^{4}$, M. Ferraro ${ }^{4}$, \\ A. Niang ${ }^{1}$, R. Crescenzi ${ }^{4}$, S. Wabnitz ${ }^{4,5}$ and V. Couderc ${ }^{3}$ \\ 12 Department of Information Engineering, University of Brescia, Via Branze 38, 25123 Brescia, Italy \\ ${ }^{3}$ Department of Physics, Chemistry and Biology, Linköping University, SE-581 83 Linköping, Sweden \\ Université de Limoges, XLIM, UMR CNRS 7252, 123 Avenue A. Thomas, 87060 Limoges, France \\ ${ }^{4}$ Department of Information Engineering, Electronics, and Telecommunications, Sapienza University of Rome, \\ Via Eudossiana 18, 00184 Roma, Italy. \\ ${ }^{5}$ Novosibirsk State University, Pirogova 1, Novosibirsk 630090, Russia \\ *fabio.mangini@unibs.it
}

\begin{abstract}
We study nonlinear self-imaging dynamics in graded-index multimode optical fibers. Side-scattering of light that accompanies the propagation of intense femtosecond pulses permits us to directly measure the self-imaging period. (C) 2020 The Author(s)
\end{abstract}

In nonlinear graded-index (GRIN) multimode (MM) optical fibers, the self-imaging phenomenon is of interest for many applications, such as implementing a fast saturable absorber mechanism in fiber lasers via multimode interference, and self-cleaning the output beam shape [1,2]. In this work, we obtain a new exact solution for the nonlinear evolution of the moments of a laser beam with a generic transverse profile, confirming that the spatial self-imaging (SSI) period does not vary with power. The longitudinal evolution of beam self-imaging is directly experimentally visualised, thanks to side-scattering of light from the fiber via second-harmonic generation, and photo-luminescence emission.

To model the beam dynamics in a MM fiber, we use the 2D nonlinear Schrödinger equation (2D-NLSE) [3]: $\frac{\partial A}{\partial z}-i \frac{1}{2 k_{0}}\left(\frac{\partial^{2} A}{\partial x^{2}}+\frac{\partial^{2} A}{\partial y^{2}}\right)+i \frac{k_{0} \Delta}{\rho^{2}}\left(x^{2}+y^{2}\right) A=i \frac{k_{0} n_{2}}{n_{c o}}|A|^{2} A$, where $k_{0}=\omega_{0} n_{c o} / c$ is the wavenumber, $\rho$ is the core radius, $\Delta=\left(n_{c o}^{2}-n_{c l}^{2}\right) / 2 n_{c o}^{2}$ is the relative index difference between the refractive index of the fiber core $\left(n_{c o}\right)$ and the cladding $\left(n_{c l}\right)$, and $n_{2}$ is the nonlinear coefficient. We assumed an infinite extension of the index profile, and disregard Raman scattering and chromatic dispersion. In our analysis we consider the beam moments as expectation values and use operator methods from quantum mechanics to derive a closed system of equations for the longitudinal evolution of the first and second order moments. In particular, we obtain a closed form solution $\left\langle x^{2}+y^{2}\right\rangle(z)=\frac{1}{4 \beta}\left\langle H_{0}\right\rangle+a_{r} \cos \left(\sqrt{\frac{8 \beta}{k_{0}}} z+b_{r}\right)$ for the radial moment associated with the rms-width for a centered beam. Here, $\left\langle H_{0}\right\rangle=\frac{1}{k_{0}}\left\langle p_{x}^{2}+p_{y}^{2}\right\rangle+2 \beta\left\langle x^{2}+y^{2}\right\rangle-\gamma\langle I\rangle$ is the Hamiltonian invariant $\left(\beta=k_{0} \Delta / \rho^{2}, \gamma=k_{0} n_{2} / n_{c o}\right)$ and $a_{r}$ and $b_{r}$ are integration constant representing the amplitude and phase of the oscillation that are determined by the initial beam profile entering the fiber. The self-imaging period $z_{s}=\pi \rho / \sqrt{2 \Delta}$ is found to depend only on the fiber parameters and to be independent of power. Unlike previous theoretical analysis that assumes the beam to maintain a specific shape during propagation through the MM fiber, this result is general and applies to the nonlinear evolution of laser beams of arbitrary transverse shape.

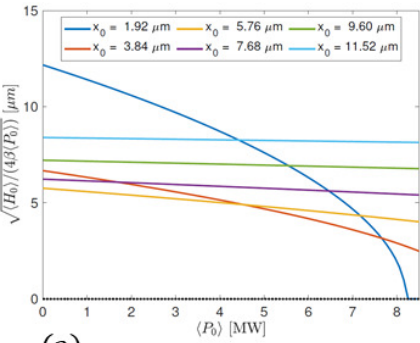

(a)

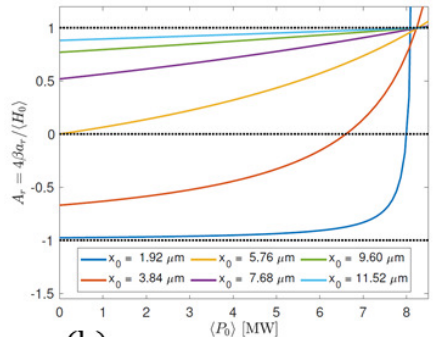

(b)

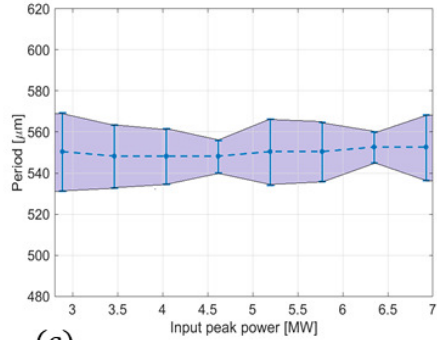

(c)

Fig. 1. Variation of (a) average beam width, (b) beam oscillation amplitude with power, and (c) experimental measurements of power dependence of self-imaging period.

In Fig. 1(a), we assume a Gaussian beam and illustrate the power-induced variation of the average beam width, i.e. $w=\sqrt{\left\langle H_{0}\right\rangle / 4 \beta\left\langle P_{0}\right\rangle}$, for different values of the input width $x_{0}$. As can be seen, the average beam width 
decreases as the power invariant $\left\langle P_{0}\right\rangle$ grows larger: the narrower the input beam size $x_{0}$, the faster the nonlinear decrease of the beam width with power. To better highlight the nonlinear dependence of the solution for the radial moment, we plot in Fig. 1(b) the input beam power $\left\langle P_{0}\right\rangle$ dependence of the beam width oscillation amplitude $A_{r}=4 \beta a_{r} /\left\langle H_{0}\right\rangle$, for different values of the input radial width $w$. As can be seen, if the initial width $w=w_{f}$, the oscillation amplitude is equal to zero in the linear limit, and it increases with power until it reaches unity for $\left\langle P_{0}\right\rangle \simeq 8.3 \mathrm{MW}$. This means that the beam width shrinks to zero, which corresponds to a beam collapse condition.

To experimentally investigate the power dependence of self-imaging in a GRIN fiber, we performed two sets of measurements with different laser sources. First, we used an ultra-short femtosecond laser system, involving a hybrid optical parametric amplifier (OPA) of white-light continuum, pumped by a femtosecond Yb-based laser, generating $70 \mathrm{fs}$ pulses at $1550 \mathrm{~nm}$ (anomalous dispersion regime), with $25 \mathrm{kHz}$ repetition rate. The input laser beam was focused by a $30 \mathrm{~mm}$ focal lens, corresponding to an input beam diameter $\left(1 / e^{2}\right)$ of $18 \mu \mathrm{m}$, into a $5 \mathrm{~cm}$ long multimode standard 50/125 GRIN fiber, with relative index difference $\Delta=0.0102$ (see results in Fig. 2, left panel). Next, we carried out measurements in the normal dispersion regime, by using a fiber laser source at 1030 $\mathrm{nm}$, generating $250 \mathrm{fs}$ pulses with a $30 \mathrm{kHz}$ repetition rate. Here we injected the pulses in a relatively short, $1 \mathrm{~cm}$ section of a 50/125 GRIN fiber (see results in Fig. 2, right panel). Fig. 2(a) shows multiple peaks of scattered light at points of peak intensity along the fiber. Spectra at the fiber output for different input peak powers (see Fig. 2(b)) show a series of dispersive wave sidebands generated from soliton oscillations. The spectrum of side-scattered light is shown in Fig. 2(c). In all spectra it is possible to note the emission of a broadband photo-luminescence, with a peak around $380 \mathrm{~nm}$. Fig. 1(c) highlights the invariance of the SSI period with power. These results confirm that the SSI period remains a constant even at the highest input peak powers.

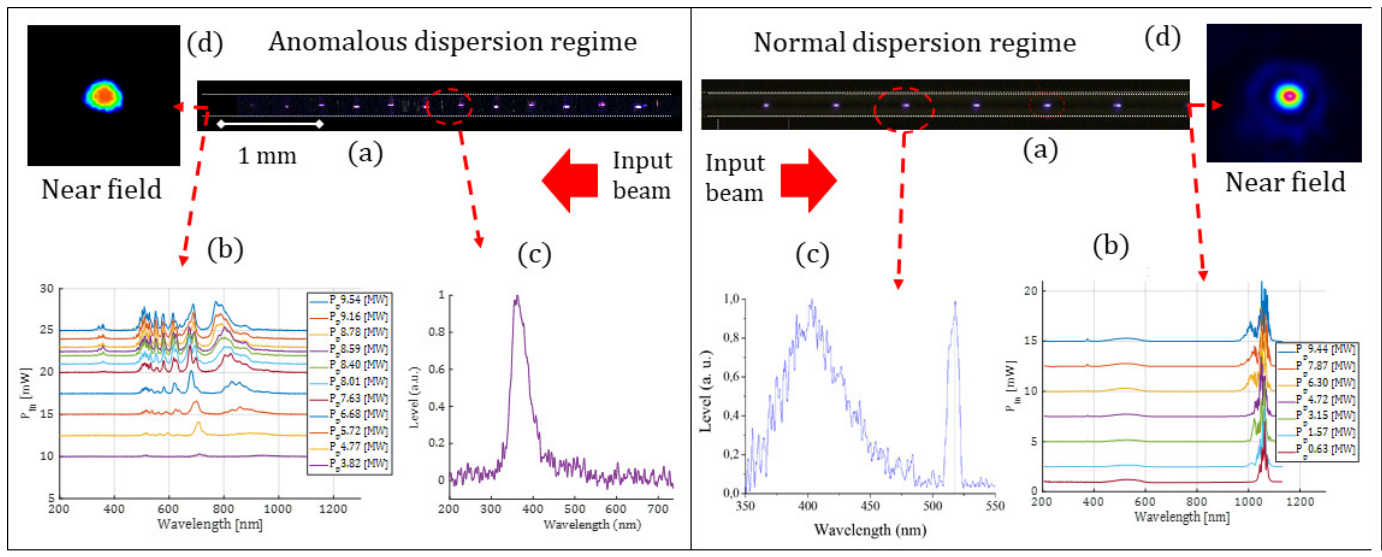

Fig. 2. Experimental results on the non-collinear frequency conversion obtained in a 50/125 GRIN fiber in anomalous dispersion (Left panel) and normal dispersion (rigth panel); (a) side image of the fiber, showing periodic emission of blue light; (b) output spectra for several input peak powers; (c) spectrum of side-scattered light; and (d) near field profile.

In summary, we studied the SSI dynamics in nonlinear GRIN-MM optical fibers. We obtained a new exact solution for describing the period and the amplitude of the beam width oscillations along the fiber. We experimentally visualized the evolution of SSI by means of ultrashort laser pulses, leading to the side-light scattering of blue luminescence and harmonic waves, confirming the invariance of the SSI period up to peak powers close to the beam collapse value.

Funding European Research Council (ERC) (740355); Agence Nationale de la Recherche (ANR) (ANR-18CE080016-01); CILAS Company (ArianeGroup) by means of the shared X-LAS laboratory; Swedish Research Council (Grant No. 2017-05309); Russian Ministry of Science and Education (14.Y26.31.0017).

\section{References}

1. E. Nazemosadat and A. Mafi, "Nonlinear multimodal interference and saturable absorption using a short graded-index multimode optical fiber," J. Opt. Soc. Am. B, 30, 1357-1367 (2013).

2. K. Krupa, A. Tonello, A. Barthélémy, T. Mansuryan, V. Couderc, G. Millot, P. Grelu, D. Modotto, S.A. Babin, and S. Wabnitz, "Multimode nonlinear fiber optics, a spatiotemporal avenue," APL Photonics, 4, 110901 (2019).

3. K. Krupa, A. Tonello, B.M. Shalaby, M. Fabert, A. Barthélémy, G. Millot, S. Wabnitz, and V. Couderc, "Spatial beam self-cleaning in multimode fibres," Nat. Photonics, 11, 234-241, (2017). 